\title{
INEXISTENCIA Y DEBILIDAD DE ACTITUDES POLÍTICAS
}

\author{
RODRIGo BaÑo \\ PRESENTACIÓN
}

Se intenta aquí abordar el difícil tema de los cambios y continuidades en actitudes y preferencias políticas en Chile, comparando el período democrático anterior al régimen militar con el momento culminante de la transición al régimen democrático que se produce a fines de los ochenta.

El estudio se centra en sólo dos aspectos: la autoubicación de los sujetos en posiciones políticas (izquierda, derecha, centro); la aproximación o preferencia partidaria.

Los datos que sirven de base a este trabajo corresponden al estudio panel realizado entre 1987 y 1989 según convenio entre FLACSO y el A. Bergerstraessen Institut de Freiburg, financiado por la Fundación Volkswagen. A su vez, la información para el período 1957-1973, pertenece a las encuestas realizadas por Mario Hamuy, recuperadas en el llamado Archivo Hamuy, elaborado también por convenio entre A.B.I. y FLACSO con el aporte de la misma Fundación Volkswagen.

\section{INTRODUCCIÓN}

A pesar del lugar central que ocupan en las ciencias sociales, los valores, actitudes y opiniones siguen presentándose como un mundo bastante misterioso y desconocido. Esto, al punto que cuestiones esenciales de ellos permanecen sin mayor aclaración. De las actitudes en general, se supone que existen y se supone que alguna relación tienen con la acción. También se supone que las actitudes pueden permanecer o cambiar. Y no es mucho más lo que se puede suponer razonablemente al respecto.

Naturalmente, no pretendemos presuntuosamente abordar el tema general de las actitudes, ni poner en discusión teorías sobre ellas. Estamos lejos de tener la capacidad para intentarlo. Sin embargo, tal vez tomando pie en estos supuestos generales sobre las actitudes, podamos organizar mejor la presentación de ciertas consideraciones que no parecen desmentidas por algunos datos.

En efecto, siendo nuestro objetivo inmediato establecer la permanencia o cambio de determinadas actitudes políticas, no podemos dejar de considerar el supuesto de la existencia de tales actitudes y la relación de ellas con la acción política. Esto no en un sentido genérico, sino en un sentido específico, vale decir, si existe o no existe una actitud formada en relación con un determinado punto y si tal actitud se expresa en algún tipo de comportamiento.

Lo anterior nos lleva también a considerar, en forma tangencial, otros problemas que han sido objeto de profunda y larga discusión en las ciencias sociales. Tal es el caso del papel que 
desempeñan elite y masa en la formacion de actitudes (Converse, 1964). También lo es la relación que hay entre individuo y sociedad en lo que se denominan actitudes sociales.

Como la permanencia o cambio de las actitudes no es considerado un hecho puramente casual, sino que provocado por cierto cambio en las condiciones en que tales actitudes existen. la hipótesis lógica sería que un cambio en las condiciones produciría un cambio en las actitudes Pero como los condicionantes de una actitud pueden ser muchísimos, no estamos en la situación de poder decir que el cambio de cualesquiera condición va a producir un cambio de actitudes. Sin embargo, cuando cambian fuertemente condiciones, que se ligan teóricamente con un determinado tipo de actitud, esperamos que ésta cambie. El estudio se dirige a confirmar si se ha producido este cambio y en que consiste.

La situación chilena de los últimos años se supone que constituye un contexto de alta probabilidad de cambio de actitudes políticas. Tal suposición se funda en el fuerte cambio que se ha producido en algunas condiciones que se consideran claves en la conformación de actitudes.

En primer lugar, se ha producido un cambio en el proceso político, puesto que ha habido una interrupción violenta y larga del régimen político democrático, con una fuerte represión hacia los partidos políticos y la participación ciudadana. La vuelta a la democracia no significa tampoco el simple retorno a la situación precedente, sino que se hace en los límites de un controlado proceso de transición.

En segundo lugar, se ha producido una importante transformación en la estructura social, alterándose la composición de los sectores que aparecían políticamente como significativos. ${ }^{1}$ En los sectores populares, disminuye la clase obrera tradicional y aumenta el sector informal o por cuenta propia. En las capas medias, el antiguo contingente ligado al aparato estatal se ve desplazado por nuevas capas vinculadas al sector servicios de tipo privado y pequeño y mediano empresariado. Tambien el empresariado sufre transformaciones merced al mayor dinamismo del sector financiero y a la mayor importancia que cobra la vinculación internacional, tanto en términos de capital como de mercados.

Por último, las transformaciones que se producen en el contexto mundial también operan en el sentido de modificar los términos ideológicos de referencia. La hegemonía del pensamiento neoliberal y el colapso de las experiencias socialistas gestadas a partir de la revolución soviética seguramente deberán influir en un país de creciente integración mundial.

Puede apreciarse que los cambios señalados son suficientemente fuertes como para esperar que ciertas actitudes políticas, especialmente las preferencias partidarias y de orientación política, hayan experimentado importantes transformaciones. A esto habría que agregar las características que muestra la transición del régimen autoritario al democrático y que. presumiblemente. pueden incidir bastante al menos en las primeras expresiones en el nuevo régimen (Baño, R. y Canales, M.. 1991).

Como se sabe, a pesar de los intentos por construir tipologías de transición, cada proceso específico tiene características peculiares difíciles de ignorar en el análisis. Al respecto, el caso chileno, de una transición tranquila y ordenada, y un modelo económico funcionando

'Fxisten varios estudios sobre cambios en la estructura social. Entre otros: Martínez y Tironi. 1985; Martinezy Leon, 1987: Campero, 1982: Baño y Faletto. 1993. 
exitosamente. es poco comparable con otras transiciones ocurridas recientemente en la región latinoamericana, y la comparación con el proceso español requeriría demasiadas precisiones.

La transición chilena parte de una especie de negociación implícita para buscar una salida política a una crisis que amenazaba transformarsc en enfrentamiento de sectores extremos. Después de un largo proceso de descompresión y apertura politica se procede a poner en funcionamiento mecanismos institucionalizados de resolución. bajo el control de las FF. AA., resultando derrotada la intención del general Pinochet de seguir al mando de la nación

Esto hace que la transición tenga un fuerte carácter de «otorgada» por el régimen militar y establezca claras limitaciones, aconsejadas por la prudencia, a las fuerzas que se oponían al general Pinochet y que finalmente triunfan en las elecciones generales de 1989.

La prudencia arranca directamente del hecho de que los antiguos sectores en el poder mantienen prácticamente toda su fuerza. Tal es el caso especialmente de las FF. AA., con un Ejército firmemente cohesionado alrededor de quien continúa siendo su Comandante en Jefe. el general Pinochet. También ocurre lo mismo en el Poder Judicial, con una Corte Suprema que no oculta sus diferencias con las nuevas autoridades. Más explícito aún fue el apoyo del empresariado al régimen del general Pinochet y mantiene esa posición sin grandes variaciones. A esto hay que agregar una derecha política, que resurge más fuerte que nunca en las elecciones generales, y que reivindica buena parte de la obra del régimen autoritario.

En suma, las características de la transición chilena y los cambios ocurridos en la situación política y social y el contexto ideológico de referencia, establecen condiciones que parecen adecuadas para producir un cambio en las actitudes políticas. Tal cambio tendría también una dirección, al menos en el sentido de una moderacion de las posiciones y un giro más hacia la derecha de todo el espectro.

Lo anterior pareciera verse corroborado por los rasgos que estarían primando en el proceso chileno, al menos a partir de los últimos años del régimen militar. Es asi como las elecciones de fines de 1989 parecieran marcar la preferencia por líderes y partidos de carácter moderado. a la vez que un concluyente centrismo politico. Hay también claramente un aislamiento de las posiciones más extremas, como en el caso del pinochetismo duro y, mucho más claramente, el Partido Comunista. A nivel del discurso se aprecia una generalizada crítica al ideologismo y el reforzamiento de posiciones.pragmáticas y conciliatorias. Entre fuerzas sociales, como trabajadores y empresarios, tradicionalmente antagónicas, se promueven y logran posiciones de consenso. En la arena política este énfasis en el consenso conduce a un creciente predominio de la administración sobre la política en gran parte de la acción gubernamental.

Por ciento que estas características del proceso chileno de comienzos de la década de los noventa, se ven permitidas o, al menos, facilitadas, por la subsistencia de un modelo económico que sigue funcionando razonablemente bien, a pesar de las críticas que puedan hacérsele desde el punto de vista distributivo. Además, salvo matices, no pareciera haber grandes diferencias entre las distintas corrientes políticas respecto del modelo económico vigente.

Frente a todos estos antecedentes casi puede parecer ocioso tratar de indagar acerca de si las actitudes políticas han cambiado o no, puesto que casi habría que concluir que forzosamente cllas han cambiado. Pero no sólo se puede alegar que cl cambio de esas condiciones no asegura un cambio de actitudes. sino que no sabemos el sentido y alcance de ese supuesto cambio, ni las expectativas de acción que se podría tener a partir de eso. Esto nos vuelve a plantear los problemas con que comenzábamos; el de la existencia de actitudes y el de su relación con la 
acción. Por cierto, todo ello dentro de límites muy estrechos, ya que nos referimos a determinadas actitudes políticas, como el posicionamiento político y las preferencias partidarias, en un ámbito espacio temporal definido: se trata de datos sobre el proceso político chileno del momento de la transición (1987-1989) en relación con datos similares del período anterior al régimen militar (1957-1973).

Es de esperar que este breve análisis logre aclarar algunos aspectos relacionados con la conformación de actitudes políticas mediante estudios comparativos.

\section{LA CONFORMACIÓN DE ACTITUDES EN LA TRANSICIÓN}

Las transiciones de regímenes políticos son normalmente entendidas como momentos cruciales en la conformación de actitudes politicas, especialmente cuando, como en el caso chileno, ha transcurrido un tiempo relativamente largo de autoritarismo político. Es de esperar que en tales situaciones existan grados elevados de ignorancia política. confusión y escasa cristalización de actitudes. Al mismo tiempo, es posible que, por recuerdo directo o a través de procesos de socialización familiar, se mantengan congelados conocimientos y actitudes generados en la experiencia democrática previa (Lipset y Rokkan, 1967). En tal caso, las expectativas son que las actitudes políticas correspondientes a la nueva situación se vayan formando progresivamente según el desarrollo del proceso político.

De ser efectivas las consideraciones precedentes, habría que pensar que durante el período de transición se plantea en forma particularmente fuerte el problema de la existencia o inexistencia de actitudes. Vale decir, el supuesto de que existen actitudes es aquí bastante difícil, independientemente que, puestos en la situación de manifestarse, los sujetos opten por determinadas preferencias. Es lo que ocurre no sólo en el caso de las encuestas de opinión. sino que, incluso, puede manifestarse en las votaciones.

Como hemos señalado en un trabajo anterior (Baño, 1989), resulta absurdo que los sujetos tengan actitudes u opiniones respecto de todo lo que se les pueda preguntar. Esta situación se refleja no sólo en los índices de las declaraciones de no saber o no contestar la pregunta, sino que en la elección de alternativas que se consideren válidas para la pregunta. Resumiendo lo presentado en ese trabajo, se puede decir que los sujetos se pueden encontrar, frente a una pregunta específica, en tres situaciones básicas: tener una actitud definida clara y consistente; no tener ninguna actitud u opinión al respecto; o tener actitudes u opiniones variables según las circunstancias.

Los sujetos que están en esta última situación son los que aportarían las mayores posibilidades de cambio a corto plazo. Los sujetos de los otros dos tipos contribuirían a la estabilidad de las actitudes: los primeros, porque la solidez de sus actitudes los hace persistir en ellas; los segundos, porque su elección de opciones será azarosa y, por tanto, se distribuirá regularmente según el cálculo de probabilidades, o simplemente no se manifestará. Por cierto que el análisis de la existencia o no de actitudes y la firmeza de estas requeriría un análisis bastante más sofisticado en cuanto a las posibilidades de cambio, pero a los efectos de este trabajo talvez baste con estas formulaciones generales.

Ahora bien, en el momento de la transición es de esperar que haya pocas actitudes y opiniones claras y consistentes en relación con muchos problemas políticos, lo cual no obsta 
a que en algunos casos haya bastante consistencia en las respuestas que se obtienen a traves de encuestas.

Esto último parece presentarse en varias cuestiones de importancia. Tal ocurre con la muy repetida pregunta respecto de opiniones sobre la democracia. En la serie de encuestas realizadas por FLACSO a partir de 1986 se observa una asombrosa estabilidad de las respuestas (ver Cuadro $\mathrm{N}^{\circ}$ l) hasta la realización de la segunda ola del estudio del panel en 1988. Sin embargo. al comparar las respuestas dadas por los mismos sujetos en la primera y segunda ola del panel se observa que hay importantes cambios, los que, al ser equivalentes en las distintas direcciones, se anulan y dan la apariencia de estabilidad. Dicho de otra manera, más provocativa, hay inestabilidad individual y estabilidad social. Paradojalmente, al momento que se produce una variación en las opiniones sobre la democracia, en la tercera ola del panel, se observa que hay un ligero aumento de la estabilidad de las respuestas individuales (Cuadros № 2 y № 3 ).

\section{Cuadro № 1}

ACTITUDES RESPECTO AL REGIMEN POLÍTICO

(PORCENTAJES)

\begin{tabular}{lcccc}
\hline & $\begin{array}{c}\text { Democracia } \\
\text { siempre }\end{array}$ & $\begin{array}{c}\text { Autoritarismo } \\
\text { algunas veces }\end{array}$ & Da lo mismo & NS/NR \\
\hline Noviembre 1985 & 57.5 & 13.3 & 25.2 & 4.2 \\
Octubre 1986 & 55.5 & 14.5 & 26.0 & 4.0 \\
Junio 1987 & 58.1 & 10.7 & 26.0 & 4.3 \\
Noviembre 1987 (Panel) & 57.4 & 13.6 & 24.0 & 5.1 \\
Agosto 1988 (Panel) & 59.0 & 11.2 & 25.6 & 4.1 \\
Noviembre 1989 (Panel) & 65.8 & 12.7 & 18.7 & 2.9 \\
\hline
\end{tabular}

\section{CuAdro № 2}

OPINIONES SOBRE DEMOCRACIA

(PoRCENTAJES, 1987/1988)

\begin{tabular}{lccccc}
\hline 1987 & Democracia & $\begin{array}{c}\text { Gobiemo no } \\
\text { democrático }\end{array}$ & $\begin{array}{l}\text { No importa } \\
\text { el réginen }\end{array}$ & NS/NR & TOTAL \\
\hline Democracia & 74.8 & 6.7 & 16.0 & 2.5 & 57.4 \\
Gobierno no democrático & 40.7 & 32.4 & 22.8 & 4.1 & 13.6 \\
No importa el régimen & 34.8 & 10.9 & 48.8 & 5.5 & 24.0 \\
NS/NR & 44.4 & 7.4 & 31.5 & 16.7 & 5.1 \\
Total & 59.0 & 11.2 & 25.6 & 4.1 & 100.0 \\
\hline
\end{tabular}


Cuadro No 3

OPINIONES SOBRE DEMOCRACIA

(Porcentajes, 1987/1988)

\begin{tabular}{lccccc}
\hline 1987 & Democracia & $\begin{array}{c}\text { Gobiemo } \\
\text { no democrático }\end{array}$ & $\begin{array}{c}1988 \\
\text { No importa } \\
\text { el régimen }\end{array}$ & NS/NR & TOTAL \\
\hline Democracia & 80.5 & 9.4 & 8.0 & 1.3 & 590 \\
Gobierno no democrático & 47.5 & 36.7 & 15.8 & 0.0 & 11.2 \\
No imponta el régimen & 43.6 & 8.1 & 42.1 & 6.2 & 25.6 \\
NS/NR & 43.2 & 22.7 & 20.5 & 13.6 & 4.1 \\
Total & 65.8 & 12.7 & 18.7 & 2.9 & 100.0 \\
\hline
\end{tabular}

No resulta aventurado sostener, a la luz de los datos revisados, que en relación a un tema que parece tan crucial, como es la preferencia por un régimen político, existe un alto número de sujetos que no tienen una actitud definida y consistente al respecto. Lo mismo parece ocurrir en relación a otros aspectos de alta relevancia en la conformación de la cultura política.

En efecto, en el estudio de panel realizado entre 1987 y 1989, se observa poco cambio en las preferencias de respuesta en los totales, no obstante lo cual se observa bastante «movimiento interior", esto es, cambios individuales que se compensan. Tal es el caso del interés por la política, actitudes proselitistas, preferencias por el cambio social, ideologías económicas, evaluación de los partidos y preferencias políticas.

Desgraciadamente no contamos con datos para hacer un análisis comparativo en relación con el período democrático anterior a 1973, puesto que tales datos sólo se pueden obtener de estudios de tipo panel como el que comentamos. Sólo podemos suponer que, dadas las características del momento de la transición, es probable que la ausencia de actitudes y opiniones formadas sea mayor en ella que en tiempos normales .

Las consideraciones precedentes no llevan a concluir que no existan actitudes o que ellas sean tan débiles que no valga la pena considerarlas. Por el contrario, las diferencias de preferencia que se producen en cada una de las preguntas nos están dando cuenta de que existen actitudes y opiniones. Lo que sí parece conveniente es relativizarlas un tanto en cuanto a la extensión social efectiva que elias tengan y a sus posibilidades de permanencia, debiendo tenerse muy presente la evolución de las tendencias en el corto plazo.

Teniendo en cuenta estas prevenciones. podemos señalar que las actitudes detectadas a través de encuestas, especialmente a través del panel que nos sirve de principal referencia. concuerdan con una imagen bastante compartida acerca de las características del proceso de transición.

En términos generales, se puede decir que existe una fuerte preferencia por formas democráticas de gobierno, una actitud reformista frente a los problemas sociales y una opción por medios institucionales y moderados de acción política. Todo esto se refuerza por un marcado centrismo en las preferencias políticas (Ver cuadros № $1,^{\circ} 4$. Nㅗ 5 y № 6 ).

Como se puede apreciar en los datos. las actitudes prevalecientes se refuerzan al avanzar la transición. Esto ocurre especialmente en la tercera ola del panel, realizada después del plebiscito en que fue derrotada la pretensión del general Pinochet de permanecer en el poder 
por ocho años más. Ese hecho pareciera ser el principal punto de inflexión dentro del proceso de transición, en el sentido de dar por concluido el régimen autoritario y ratificada la definitiva transición a la democracia.

\section{Cuadro № 4}

ACTITUDES HACLA EL CAMBIO SOCLAL

(PANel, PORCENTAJEs )

\begin{tabular}{lrrr}
\hline & 1987 & 1988 & 1989 \\
\hline Cambio revolucionario & 9.9 & 7.6 & 5.3 \\
Reformas graduales & 68.5 & 72.2 & 77.1 \\
Ningún cambio & 13.6 & 14.5 & 14.0 \\
NS/NR & 8.0 & 5.7 & 3.6 \\
\hline
\end{tabular}

\section{Cuadro № 5}

FORMAS DE ACCIÓN POLITICA

(Porcentajes Panel. 1987)

\begin{tabular}{lccc}
\hline & Aprueba & No aprueba & NS/NR \\
\hline Acudir a las autoridades & 69.6 & 25.9 & 4.5 \\
Recurrir a tribunales de justicia & 67.6 & 26.3 & 6.1 \\
Petición con firmas & 62.5 & 30.7 & 6.7 \\
Circulares o volantes & 33.6 & 59.2 & 7.1 \\
Marcha para reclamar & 40.2 & 54.4 & 5.4 \\
Paros parciales & 32.6 & 61.6 & 5.8 \\
Corar el tránsito & 14.8 & 79.7 & 5.5 \\
Ocupar oficinas de gobiemo & 15.3 & 78.1 & 6.7 \\
Dañar edificios públicos & 3.1 & 92.5 & 4.4 \\
Paro Nacional & 44.0 & 49.8 & 6.2 \\
\hline
\end{tabular}

\section{Cuadro $N^{\circ} 6$}

AUTOUBICACIÓN EN EL EJE IZQUIERDA - DERECHA

(PANEL, PORCENTAJES)

\begin{tabular}{lrrr}
\hline & 1987 & 1988 & 1989 \\
\hline Izquierda (1-2) & 9.5 & 9.6 & 7.8 \\
Centroizquierda (3-4) & 11.6 & 14.2 & 17.0 \\
Centro (5-6) & 32.1 & 35.5 & 39.6 \\
Centroderecha (7-8) & 9.6 & 9.5 & 12.7 \\
Derecha (9-10) & 7.7 & 7.2 & 6.7 \\
NR/Otros & 29.4 & 24.0 & 16.2 \\
& & & \\
Total & 100.0 & 100.0 & 100.0 \\
\hline
\end{tabular}


Sin embargo, hay que hacer notar que, comparativamente con otros países, en Chile el apoyo a la democracia no pareciera ser tan rotundo, sino que subsiste un sector minoritario pero importante que se declara indiferente al régimen politico o acepta la legitimidad de un régimen autoritario. Esto ratifica el componamiento electoral, tanto en el plebiscito de 1988 como en las elecciones generales de 1989, donde el general Pinochet y la derecha política que lo apoyó obtuvieron cerca de un $43 \%$ de los votos. Es decir, aunque minoritario, el régimen militar parece haber tenido un importante apoyo popular. Situación esta última que puede haberse visto reforzada, al concluir el gobierno autoritario, por el reconocido éxito económico que finalmente estaría presentando el modelo duramente aplicado por los militares encabezados por el General Pinochet, que se confió a los tecnócratas neoliberales.

Lo anterior pareciera corresponderse con otros datos del mismo momento, que revelan un bajo interés por la política bastante persistente. y una imagen negativa de los partidos políticos (Ver Cuadros No 7 y $N^{\circ} 8$ ).

\section{Cuadro № 7}

INTERÉS POR LA POLITICA*

\begin{tabular}{lccccc}
\hline & Mucho & Bastante & Poco & Nada & NS/NR \\
\hline Noviembre 1985 & 33.3 & & 31.5 & 33.5 & 1.7 \\
Octubre 1986 & 18.8 & & 34.3 & 46.0 & 1.6 \\
Junio 1987 & 23.2 & & 41.3 & 34.6 & 1.0 \\
Octubre 1987 & 20.6 & & 37.3 & 41.1 & 0.9 \\
Noviembre 1987 (Panel) & 9.3 & 21.6 & 33.8 & 34.5 & 0.7 \\
Agosto 1988 (Panel) & 9.4 & 17.3 & 33.5 & 38.9 & 0.9 \\
Noviembre 1989 (Pane1) & 9.2 & 22.7 & 37.3 & 30.5 & 0.4 \\
1969 & 12.6 & 27.6 & 22.2 & 35.7 & 2.0 \\
\hline
\end{tabular}

* Los datos de 1985 en adelante corresponden a encuestas realizadas por FLACSO en las fechas que se indican. Los de 1957 a 1973 son de encuestas realizadas por Eduardo Hamuy. En el primer caso están publicadas en los informes de investigación correspondientes; en el segundo están en el Archivo Hamuy elaborado a partir de los datos originales, segün convenio con el A. Bergerstraessen Institut de Freiburg, financiado por la Fundación Volkswagen.

CUADRO N² 8

IMAGEN DE LOS PARTIDOS

(PorCENTANES)

\begin{tabular}{lccc}
\hline & 1987 & 1988 & 1989 \\
\hline Los partidos solo sirven para dividir (Acuerdos) & 52.4 & 53.5 & 53.0 \\
Los paridos son todos iguales (Acuerdos) & 45.5 & 48.8 & 48.1 \\
\hline
\end{tabular}

Podemos modificar entonces un poco la primera impresión de que hay actitudes claramente favorables a la democracia. al reformismo y a la moderación. señalando que es bastante probable que exista una elevada proporción de personas que no tiene una actitud definida al 
respecto. A la vez, parece haber una tendencia a la apatía o enajenación del proceso político que puede dificultar el hecho de que los sujetos vayan adquiriendo actitudes consecuentes con el desarrollo del régimen democrático.

Si efectivamente no existen actitudes definidas respecto a importantes cuestiones políticas ( $y$ hay fuertes resabios de autoritarismo político) no se plantea necesariamente una amenaza a la estabilidad del proceso político, pues, como se mostrará más adelante, ésta puede descansar más en la apatía política que en la movilización. Sin embargo, bajo tales circunstancias, puede profundizarse la tendencia a la autonomización de la política de la sociedad, lo cual implica modificaciones importantes respecto del carácter y funciones del sistema político, cuyas consecuencias son difíciles de predecir.

Por otra parte, esta situación de relativa apatía y enajenación política no tiene por qué ser necesariamente novedosa ni atribuible a cambios provocados por el largo período de autoritarismo. Aunque, como ya señalamos, no tenemos datos adecuados para medir la consistencia que tuvieron las actitudes políticas en el régimen democrático anterior al golpe militar de 1973 , hay algunos indicios que permiten pensar que ciertos rasgos de apatía política y cuestionamiento de los partidos se presentaron ya en el pasado.

En el año 1969 se hizo una pregunta casi idéntica a la del panel sobre interés por la política y. a pesar de que era un momento de alta movilización política. inmediato a las elecciones parlamentarias y a un año de las determinantes elecciones presidenciales, se obtiene sólo un poco más de interés por la política que en los años 1987, 1988 y 1989 (Cuadro № 7). A la vez, la favorabilidad a los partidos es tan baja en el período reciente como desde 1958 a 1973. con claro predominio de los «en contra» por sobre los «a favor». Con la excepción, antes y ahora, de la Democracia Cristiana, que es la única que eventualmente logra más de la mitad con los que la favorecen (Cuadro $\mathrm{N}^{\circ}$ 9).

Por otra parte, ante la pregunta más dura sobre los partidos, esto es, si son necesarios (para gobernar o para defender los intereses de grupos y clases) es posible encontrar desde 1964 una recalcitrante cuarta parte de sujetos que simplemente declaran que no son necesarios (Cuadro №10).

Todo esto pareciera demostrar que no resulta tan sencillo concluir que las actitudes han cambiado y que, en consecuencia, es posible esperar el desarrollo de un proceso bastante distinto al del pasado. Incluso puede encontrarse un asombroso parecido en la posición que se tenía de la legalidad del Partido Comunista en 1958 y la que se tiene en el período 1987-1989 (Cuadro $N^{\circ} 11$ ). Esto a pesar de que se suele considerar que una de las actitudes que más claramente ha cambiado se relaciona con el rechazo al Partido Comunista.

En todo caso, estas comparaciones aisladas no resultan fáciles de interpretar, aunque sí nos sirven para relativizar conclusiones precipitadas acerca de la novedad de la actual situación. Tal vez si tratamos de estudiar el desarrollo de actitudes. como proceso a través de un período relativamente largo, podamos aventurar algunas hipótesis descriptivas de la actual situación. Para ello nos centraremos, por requerimientos de la brevedad y de las restricciones de información que manejamos, a la autodefinición de la posición política de los sujetos, así como, en ciertos casos, a las preferencias políticas directas. La comparación es entre el período de aproximadamente quince años antes del régimen militar y el de los años claves de la transición estudiados en el panel a que hacemos referencia. 
REVISTA DE SOCIOLOGIA

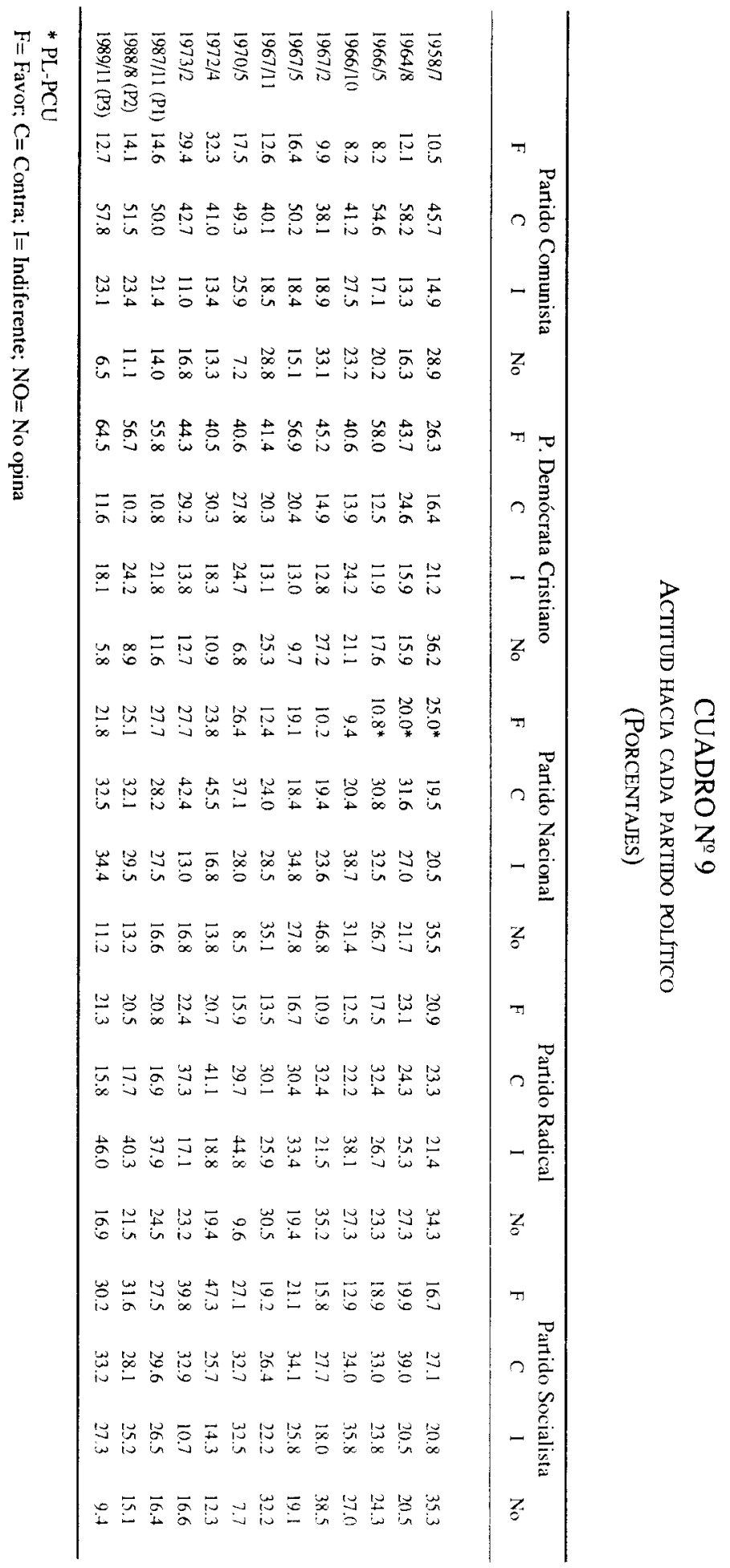


CUADRO N ${ }^{2} 10$

NECESIDAD DE LOS PARTIIDOS

(PARA GOBERNAR HASTA 1973; PARA DEFENDER INTLRESES DE GRUPOS Y ClASFS EN 1987-1989)

\begin{tabular}{lrrrrrrrrrrr}
\hline & 1964 & $1965 / 1$ & $1970 / 5$ & $1970 / 7$ & $1972 / 4$ & $1972 / 12$ & $1973 / 2$ & $1987 / 11$ & $1988 / 8$ & $1989 / 11$ \\
\cline { 6 - 13 } Vecesarios & 62.4 & 70.7 & 64.7 & 65.0 & 73.3 & 72.8 & 68.4 & 69.8 & 71.5 & 71.7 \\
Vonecesarios & 24.3 & 20.1 & 30.8 & 30.6 & 21.6 & 24.2 & 26.5 & 20.6 & 19.8 & 21.0 \\
VSNR & 13.3 & 9.2 & 4.6 & 4.4 & 5.1 & 3.1 & 5.0 & 9.6 & 8.7 & 7.3 \\
\hline
\end{tabular}

CUADRO № 11

Partido Comuntsta FUERA de l.A LEY

(PoRCENTAJES)

\begin{tabular}{lrrrr}
\hline & 1958 & 1987 & 1988 & 1989 \\
\hline Acuerdo & 37.2 & 35.5 & 38.9 & 38.1 \\
Desacuerdo & 53.7 & 49.9 & 49.0 & 53.5 \\
XS/NR & 9.2 & 14.6 & 12.1 & 8.4 \\
\hline
\end{tabular}

\section{LAS POSICIONES POLÍTICAS}

Normalmente en Chile, como en otros países, se le ha atribuido gran importancia a la ubicación en el espacio político que corresponde al eje izquierda-derecha. Se podría decir que se presenta como el principal eje ordenador de organizaciones, ideologías, programas, políticas y sujetos. Más aún, el sistema político partidario tuvo siempre una referencia explícita y directa con este eje ordenador.

El conocimiento de este espacio político pareciera estar probado en la tradición política chilena. A la vez, en las encuestas realizadas durante el proceso de transición, es posible observar una asombrosa precisión en la ubicación que los entrevistados asignan a los partidos políticos, antiguos y nuevos, que emergen a la luz pública (Cuadro № 12). Naturalmente se aprecia un menor conocimiento de los partidos nuevos. como es el caso de Renovación Nacional, creada a partir de algunos elementos del antiguo Partido Nacional e independientes de derecha; y Avanzada Nacional, un partido creado prácticamente como aparato de apoyo del general Pinochet cuando resurgen los partidos políticos. Pero, aún en estos casos, se puede apreciar que son bien ubicados, incluso en las siguientes olas del panel (1988 y 1989), al aumentar el conocimiento de estos partidos. aumenta la precisión con que son ubicados. Situación que, por lo demás, ocurre con respecto de todos los partidos. Algo similar sucede con otros partidos nuevos, como el PPD (creado por el socialismo renovado) que se incorpora en la segunda ola y la UDI (desmembrada de Renovación Nacional, después de haber concurrido a su formación) y el PAIS (creado con fines electorales por comunistas y socialistas ortodoxos).

Por otra parte. el eje izquierda derecha posee una gran capacidad discriminatoria en relación con aspectos de cultura política. tal cual ocurre en los sistemas políticos de Europa 
continental (Sartori, 1980; Baño, 1990). Lo cual ratifica la importancia del posicionamiento en el espacio político.

\author{
CUAadro No 12
}

UBICACIÓN DE LOS PARTIDOS EN EL EJE IZQUIERDA-DERECHA

(Nov. 1987, PorCENTAJES)

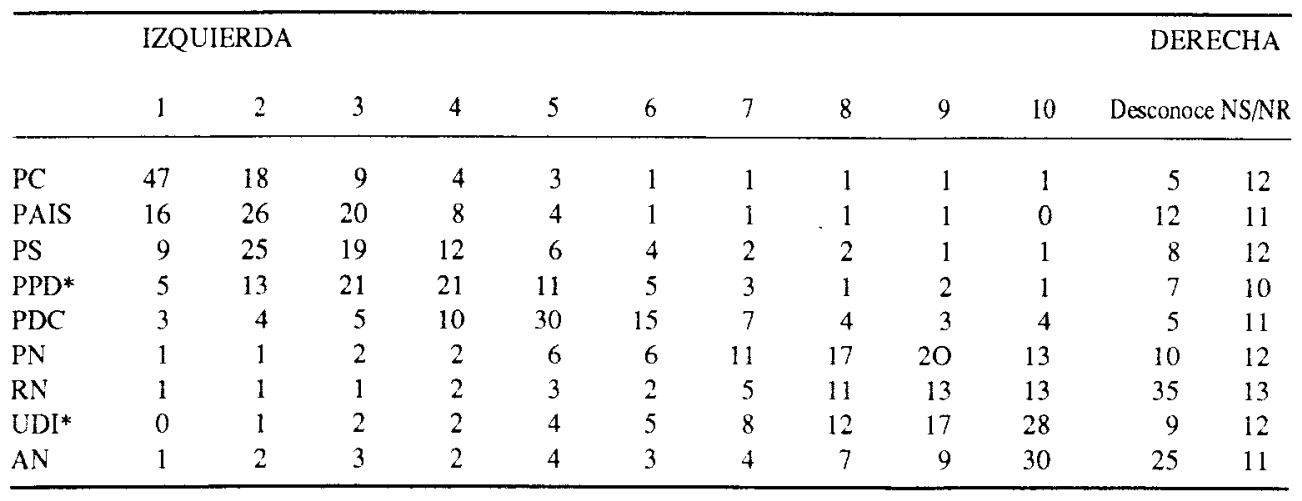

Los partidos PAIS, PPD y UDI tienen la ubicación que le dieron los entrevistados en la tercera ola (1989), ya que antes no se les incluyó por no existir.

En el cuadro los partidos se ubicaron de arriba a abajo desde el más de izquierda, el comunista, al más de derecha, Avanzada Nacional.

No obstante, es sabido que el significado del posicionamierto político resulta bastante oscuro. Es difícil determinarlo conceptualmente y más difícil aún es saber el sentido que tiene para distintos sujetos en distintos espacios y tiempos. Más aún en las actuales circunstancias, de quiebre de las posiciones socialistas que pone en cuestión los alcances de lo que podría ser un continuo ideológico entre capitalismo y socialismo. La repetida pregunta del momento: ¿qué significa hoy ser de izquierda? da buena cuenta del problema. Reducirlo a un continuo programático no facilita las cosas, ya que un programa tendría que tener una referencia ideológica para poder ser ubicado posicionalmente.

En todo caso, es posible tratar de establecer si en el proceso político chileno, el posicionamiento en el eje izquierda derccha se relaciona o no con algunos elementos que desde la perspectiva de ese proceso. considerado especificamente a partir de los años cincuenta, se le asignaban. Esto, sin entrar en el análisis conceptual de los términos.

Se podrían señalar dos elementos de carácter programático y uno de representación social. En primer lugar, se asigna a las posiciones de izquierda una mayor predisposición al cambio frente a una derecha más conservadora. En segundo lugar, la izquierda es considerada, desde el punto de vista económico, en una disposición más estatista que la derecha. Estos elementos de carácter programático no tienen un carácter absoluto, pero parecieran corresponder con las tendencias observadas en el discurso político desde que se establecen las diferencias izquierda y derecha en Chile. Es la razón de que sean tomados como referencia para tratar de establecer alguna relación de contenido en el posicionamiento político de los sujetos. 
En cuanto a la representación social que pudiera implicar la diferenciación izquierda Jerecha. ella arranca del origen mismo del sistema de partidos modemos (Cerroni, 1971), ya que los primeros partidos surgen para representar una parte de la sociedad, trabajadores, que no se considera representada en quienes detentan el poder en nombre de la razón general. Los partidos socialistas, y de izquierda en general, se atribuyen la representación de esos sectores populares. En Chile, el nacimiento de los partidos de izquierda, de clara orientación marxista. tiene explícitamente la misma pretensión de representación social.

Respecto al tema de la representación social, hay en Chile interesantes trabajos que tienden a demostrar que existen relaciones entre votación de los partidos de izquierda y sectores populares (Faletto, 1988; Aldunate, 1985; Baño, 1990). Sin embargo, eso no nos soluciona el problema de la relación entre la ubicación en el espacio político y sectores sociales definidos especialmente en términos de estratificación.

Lo datos existentes de encuestas de opinión pública antes del quiebre institucional nos señalan que, relativamente, pareciera haber cierta relación entre posicionamiento político y los clementos que usualmente se han vinculado a éste.

Respecto del elemento cambio, resulta bastante difícil establecer la relación, debido a que la pregunta genérica sobre necesidad de cambios realizada en 1964 y en 1965 encuentra muy pocos entrevistados que se manifiestan contrarios a ellos. En todo caso, es perceptible. especialmente en el año 1964, una mayor aceptación del cambio en la izquierda que en la derecha (Cuadro № 13 ). Esta relación pareciera mantenerse en el momento de la transición, 1987-1989, ya que, tanto ante preguntas específicas como frente a la pregunta genérica sobre cambio social la izquierda aparece apoyando más los cambios y en forma más radicalizada , Cuadro № 14).

CuAdro N"13

NeCESIDAD de CAMBIOS SOCIALES SEGÚN POSICIÓN POLITICA (Porcentajes)

\begin{tabular}{lll}
\hline & 1964 & 1965 \\
\hline Derecha & 55.4 & 77.6 \\
Centro & 70.3 & 85.8 \\
Izquierda & 79.2 & 85.7 \\
\hline
\end{tabular}

Cuadro No ${ }^{2} 14$

ACTITUDES FRENTE AL CAMBIO SEGUN POSICIÓN POLITICA

(Porcentajes Panel 1987)

\begin{tabular}{lcccc}
\hline & Revolucionario & Reformista & Conservador & NS/NR \\
\hline Izquierda & 24.7 & 68.0 & 4.4 & 3.1 \\
Centro & 5.0 & 80.1 & 12.3 & 2.6 \\
Derecha & 7.1 & 63.0 & 26.6 & 3.3 \\
\hline
\end{tabular}


En cuanto al estatismoeconómico, la relación también pareciera ser clara, tanto en estudios hechos en 1961 y 1964 , como en el panel reciente (Cuadro $N^{\circ} 15$ y $\mathrm{N}^{\circ} 16$ ).

\section{Cuadro N" 15}

FaVORABILIDAD HaCla ESTADO PROPIETARIO

(PorcentaJes De CADA TENidenCla)

\begin{tabular}{lcc}
\hline & 1961 & 1964 \\
\hline Derecha & 32.0 & 26.8 \\
Centro & 27.3 & 15.8 \\
lzquierda & 52.3 & 41.9 \\
\hline
\end{tabular}

\section{Cuadro No 16}

CONTROL DE LAS INDUSTRIAS SEGUN POSICIÓN POLITICA (Porcentajes Panel 1987)

\begin{tabular}{lccc}
\hline & Privado & Estatal & NS/NR \\
\hline Izquierda & 39.1 & 55.1 & 5.8 \\
Centro & 55.8 & 39.5 & 4.7 \\
Derecha & 59.8 & 33.2 & 7.1 \\
\hline
\end{tabular}

En cambio. la relación entre posición política y estratificación económica podría ser mucho más compleja. contradiciendo la aparentemente simple y permanente vinculación entre votación de partidos de izquierda y sectores populares. Evidentemente puede existir cierto margen de error en los cálculos, puesto que en algunos casos se estratificó por ingreso y en otros por la evaluación que hace el entrevistador de la situacion económica del entrevistado tomando en cuenta una serie de antecedentes (barrio, calidad de la vivienda, artefactos electrodomésticos, presentación personal. etc.). Pero el margen de error no puede ser muy elevado, ya que en una encuesta en que se hizo los dos tipos de mediciones se encontró una correlación entre ellas de Gamma .734. lo que estaría demostrando que con ambos datos se está midiendo practicamente lo mismo. Además que la tendencia general persiste en el caso de encuestas con la misma medición.

Para posición política y estratificación tenemos abundantes datos, los que fueron trabajados cstadísticamente en términos de tres estratos sociocconómicos que. desgraciadamente. no pudieron ser exactamente iguales, ya que los tramos de ingreso no constituían un continuo divisible en cualquier punto. A la vez. la posición política es considerada en los tres puntos básicos de derecha. centro e izquierda.

Los resultados obtenidos resultan particularmente interesantes. En términos simples. comparando el mismo coeficiente de correlación gamma (Cuadro $\mathrm{N}$ 17) tenemos una clara evolución. que va desde una baja y casi nula relación entre ingreso y posición política en los años 1961-1966-1969 y principio de 1970. que empieza a incrementarse a partir de mediados de ese año para llegara an fueric nivel de 28 en abril de 1972. En las dos últimas encuestas decac 
un tanto el índice de correlación, pero cabe advertir que, por los problemas señalados de los datos, se altera la relación entre sectores altos y bajos en desmedro de estos.

La fuerza de este deslizamiento hacia la izquierda de los sectores más bajos es posible apreciarla más claramente aún cuando se observa la forma en que se ubica en el espacio político el tercio de más bajo nivel económico de la población. Ese sector va desde definición de poco más de un quinto en la izquierda a comienzo de los sesenta hasta más de la mitad en esa posición durante el gobierno de la Unidad Popular. (Cuadro № 18 ).

No pareciera existir aquí un efecto de apoyo popular al poder establecido, puesto que en 1961, bajo el gobierno de derecha de Jorge Alessandri, no hay un fuerte apoyo popular a la derecha; y en 1969, con el centrista gobierno de la Democracia Cristiana, no hay un fuerte apoyo al centro. La identificacion de los sectores populares con la izquierda pareciera estar dando cuenta de una creciente identificación social en el conflicto político planteado bajo el gobierno de Salvador Allende. Las ideologías, presentadas en términos de lucha de clases tradicionalmente por la izquierda chilena, podrían tener en esos momentos una traducción social efectiva, aunque, vale la pena recordar, aquí es perceptible en términos del corte ricos pobres más que en los de clase obrera o trabajadora que correspondía a la doctrina ortodoxa.

\author{
CuAdro № 17 \\ EVoluCión dEL COEFICIENTE dE CORRELACIÓN GAMMA \\ ENTRE ESTRATIFICACIÓN SOCIOECONÓMICA Y POSICIÓN POLITICA
}

\begin{tabular}{ll}
\hline 1961, julio & .057 \\
1966, mayo & .060 \\
1966, mayo & .018 \\
1969, julio & .042 \\
1970 , marzo & .055 \\
1970 , mayo & .158 \\
1970, julio & .182 \\
1970, agosto & .155 \\
1977, abril & .280 \\
1972, diciembre & .110 \\
1973, febrero & .101 \\
\hline 1987, noviembre & $.12 *$ \\
1988, agosto & .08 \\
1989, noviembre & .06 \\
\hline
\end{tabular}

\footnotetext{
* El coeficiente se ve fuertemente aumentado en 1987 y, también en 1988 debido a que el tramo de menor ingreso abarca el $64 \%$ y el $55 \%$ de la población, respectivamente.
} 
Cuadro No 18

AUTOUBICACIÓN DEL SECTOR MÁS POBRE*

\begin{tabular}{lllll}
\hline & Derecha & Centro & Izquierda & NS/NR \\
\hline $1961 / 7$ & 21.6 & 45.4 & 21.6 & 11.3 \\
$1966 / 5$ & 17.4 & 44.0 & 24.5 & 14.1 \\
$1969 / 4$ & 16.4 & 27.9 & 31.1 & 24.6 \\
$1970 / 3$ & 21.7 & 19.6 & 31.5 & 20.8 \\
$1970 / 5$ & 23.8 & 20.7 & 36.3 & 19.2 \\
$1970 / 7$ & 20.8 & 25.2 & 46.0 & 8.0 \\
$1970 / 8$ & 23.0 & 18.4 & 36.7 & 22.0 \\
$1972 / 4$ & 10.1 & 22.0 & 57.5 & 10.5 \\
$1972 / 12$ & 24.8 & 19.2 & 41.6 & 14.4 \\
$1973 / 2$ & 16.0 & 17.8 & 50.1 & 7.5 \\
$1987 / 11(\mathrm{Pl})$ & 16.1 & 27.5 & 22.4 & 33.8 \\
$1988 / 8(\mathrm{P} 2)$ & 15.2 & 31.0 & 23.5 & 30.3 \\
$1989 / 11(\mathrm{P} 3)$ & 17.7 & 36.3 & 24.5 & 21.5 \\
\hline
\end{tabular}

* Hasta 1973 se considera aproximadamente el 30\% como el sector más pobre. En el panel, al mantenerse sólo tres tramos de ingreso en valor nominal, hay variaciones debidas fundamentalmente a la inflación. En 1987 el tramo más pobre considerado es el $64 \%$, en 1988 es el $55 \%$ y en 1989 es el $40 \%$.

Cuando se compara esos datos con los correspondientes al estudio de 1987-1989 es posible observar que la situación ha cambiado radicalmente. Desgraciadamente, no es posible apreciar diferencias en el posicionamiento político de los tres estratos económicos en que se han clasificado los entrevistados debido a los cambios en las proporciones que corresponden a los tres estratos de ingreso usados en el panel. Esto es particularmente notorio en 1987, cuando el estrato más pobre abarca cerca de dos tercios de entrevistados, mientras en 1988 corresponde al 55\% y en 1989 el 40\%. Los coeficientes Gamma se ven bastantes afectados por este hecho. No obstante. incluso en estas condiciones el coeficicnte Gamma correspondiente a 1988 y 1989 ratifica la inexistencia de correlación entre ambas variables. La comparación de la distribución en el cspacio político del segmento más pobre muestra con más claridad la baja proporción de sujetos que se ubica en posiciones de izquierda. En suma, encontramos que, a nivel de encuestas de opinión pública, la situación socioeconómica no incidiría significativamente en la posición política. La atribución de representación social de la izquierda, genéricamente entendida, no pareciera tener bases sólidas al momento de la transición.

Puede apreciarse así que de los elementos que se han vinculado al posicionamiento político. los dos que son de contenido programático parecieran corresponder en términos generales y aproximativos al posicionamiento político tanto antes del régimen militar como en el momento de su transición. En cambio, el elemento representación social sólo pareciera tener vigencia. y fuertemente, durante el período de la Unidad Popular. Como veremos más adelante, esto no es un dato aislado, sino que parece corresponder a características muy notables del proceso político de ese momento.

En efecto, si revisamos, a través de datos de encuesta, el desarrollo del posicionamicnto político de la población de Santiago, veremos que nuevamente encontramos notoriamente marcada la época de la Unidad Popular en términos de polarización izquierda derecha. Esto 
pareciera demostrar que la polarización política de entonces no era, como algunos pretendían. un problema sólo de dirigencias, sino que permeaba a prácticamente toda la sociedad. Se puede constatar(Cuadro № 19) el fuerte aumento que experimenta la posición de izquierda entre 1970 y 1973 y, al mismo tiempo, la disminución de la no respuesta. Se puede medir la agudización del conflicto al constatar que prácticamente desaparecen los «neutrales».

Pero, más allá de estas connotaciones respecto al período 70-73. esta larga serie de autoubicación política nos da cuenta también de otros hechos notables que tienen que ver con formas más complejas de definición de la política. Dos de estos parecieran operar en la misma direccion de alienación política que nos parece oportuno resaltar.

CUadro № 19

AutOUBiCACIÓN EN EL ESPACIO POLITTCO

(PORCENTAJES)

\begin{tabular}{lllll}
\hline & Izquierda & Centro & Derecha & NS/NR Otros \\
\hline $1957 / 10$ & 15.1 & 8.2 & 18.3 & 58.5 \\
$1958 / 7$ & 24.3 & 17.7 & 31.4 & 26.6 \\
$1961 / 7$ & 26.6 & 28.2 & 23.7 & 21.5 \\
$1964 / 8$ & 23.4 & 23.6 & 15.0 & 38.1 \\
$1965 / 1$ & 28.4 & 29.1 & 20.8 & 21.6 \\
$1966 / 5$ & 21.5 & 31.3 & 18.7 & 28.5 \\
$1967 / 11$ & 33.3 & 21.8 & 21.1 & 23.8 \\
$1969 / 2$ & 28.3 & 27.8 & 22.0 & 21.9 \\
$1969 / 4$ & 28.1 & 32.0 & 16.5 & 23.4 \\
$1969 / 7$ & 27.2 & 26.8 & 23.6 & 21.4 \\
$1970 / 3$ & 26.1 & 24.3 & 26.6 & 23.1 \\
$1970 / 3$ & 29.2 & 23.2 & 23.3 & 24.5 \\
$1970 / 5$ & 29.9 & 26.4 & 26.1 & 17.7 \\
$1970 / 7$ & 36.0 & 30.8 & 22.2 & 10.9 \\
$1970 / 8$ & 32.7 & 15.7 & 23.7 & 17.9 \\
$1972 / 4$ & 48.7 & 25.8 & 14.9 & 10.7 \\
$1972 / 12$ & 36.9 & 30.8 & 23.2 & 9.2 \\
$1973 / 2$ & 42.8 & 26.8 & 21.8 & 8.7 \\
$1987 / 8($ P1)* & $21.1(1-4)$ & $32.1(5-6)$ & $17.2(7-10)$ & 29.4 \\
$1987 / 11$ (P2)* & $23.8(1-4)$ & $35.5(5-6)$ & $16.7(7-10)$ & 24.0 \\
$1989 / 8($ P3)* & $24.7(1-4)$ & $39.6(5-6)$ & $19.4(7-10)$ & 16.2 \\
\hline
\end{tabular}

* En 1987, 1988 y 1989 se agruparon los valores del eje de 1 a 10 en que se autoubicaron los sujetos de izquierda a derecha.

Uno de ellos se refiere a que es fácilmente perceptible que en vísperas de una elección presidencial (julio de 1958, agosto de 1964 y agosto de 1970) hay un claro aumento de aquellos in ninguna definición en el espacio político. Es decir, enfrentados a tener que definirse en un tiempo de decisión política clave, un buen porcentaje de sujetos pareciera preferir «agacharse» para esperar saber primero quien gana.

El otro hecho es que, también antes de la elección presidencial hay un incremento en las posiciones que tienen posibilidades de éxito en la competencia: izquierda y derecha en 1958 . ientro e izquierda en 1964, izquierda y derecha en agosto de 1970. Lo mismo se podría decir 
del crecimiento desmesurado del centro en 1989. aunque hay que advertir que a la inversa de lo que ocurría antes, aqui hay una disminución de los que no se definen. Estos dos hechos parecieran dar cuenta de una especic de «oportunismo político» de un sector importante que carece de definición previa y que aparece dispuesto a apoyar al ganador. Conducta que no tienc por qué ser considerada irracional. pues puede descansar en la convicción de que la política es: importante pero ajena. en cuyo caso es mejor estar con los que triunfan que con los que pierden. Es la antigua noción del voto útil. Naturalmente en momentos que se agudiza el conflicto y aumenta el compromiso político, como ocurre en el periodo 70-73, disminuye la disponibilidad de este «voto útil».

Comparado con el período anterior al golpe militar, en la transición se produce un realineamiento en que resalta la hipertrofia del centro, que llega a niveles nunca alcanzados. siendo el porcentaje más próximo el que tiene en 1965-1966, años de euforia en que Frei gana la presidencia con mayoría absoluta y la Democracia Cristiana arrasa en el Congreso.

Los datos de posicionamiento político parecen tener fundamental importancia desde la perspectiva de la aqudización o reducción del conflicto político. Desde tal perspectiva se corresponden con el diferente clima imperante en el Gobiemo de la Unidad Popular y el existente en el momento de la transición. En el mismo sentido, parecieran revelar también el mayor o menor grado de compromiso o involucramiento político con mayor precisión que la pregunta sobre interés en la política. Esto se aprecia especialmente en el pancl realizado durante la transición, comparando la escasa variación que experimenta en las tres olas el interés por la política, con el fuerte descensode la no clasificación en el espacio político. Es decir, puede que la política no interese más al fin de la transición, pero la gente va comprometiéndose con posiciones.

La gran diferencia que existe entre esta disminución de no clasificados, comparada con la que ocurre en el proceso previo a 1973, está tanto en las diversas proporciones de no clasificados, como en la dirección en que se mueve esa disminución. Aunque el porcentaje de no clasificados disminuye desde 1987 a 1989 a casi la mitad, todavía esta cifra es el doble que la obtenida en 1973. Además, y quizás sea lo más importante, a diferencia de la disminución de no clasificados en los setenta. aquí esa disminución se dirige fundamentalmente al centro. puesto que derecha e izquierda prácticamente se mantienen. Se trata, pues. de un compromiso que no agudiza el conflicto, sino que lo distiende.

Pero, a pesar de la importancia que pareciera tener el posicionamiento político. especialmente como eje ordenador del sistema de partidos y referente del carácter del conflicto político. según hemos señalado, presenta el problema de su gran relatividad. Situación que posiblemente arranca del hechocomentado de que carece de unadefinición precisa traducible operacionalmente y que no constituye tampoco una organización que sirva de referente, como es el caso de los partidos políticos.

Talvez eso hace que, en general, encontremos proporciones relativamente altas de la población que se nieguen a clasificarse en el espacio político, incluso cuando cllo se hace a través de artefactos atractivos como es el ya clásico dibujo del cje izquierda derecha para que cl entrevistado se ubique. Además, como se demuestra en el panel, se produce un alto grado de cambio de posiciones interiores en las distintas olas. lo que da cuenta no sólo de tendencias propias de un proceso político, sino que de una gran inestabilidad. Aun cuando las diez. posiciones originales se agrupen estadísticamente en cinco, para cancelar los cambios entre dos puntos inmediatos, la estabilidad de las respuestas sólo a al anza a aproximadamente un tercio. 
Por otra parte, y talvez en relación muy directa con lo anterior, no existe una buena relación entre los datos de posicionamiento en el eje izquierda derecha y los resultados electorales correspondientes al momento (Cuadro № 20)

Cuadro № 20

COMPARACIÓN dE UBICACIÓN CON VOTACIÓN EFECTIVA DE TENDENCIAS

(PORCENTAJES)

\begin{tabular}{lcccccc}
\hline & \multicolumn{2}{c}{ DERECHA } & \multicolumn{2}{c}{ CENTRO } & \multicolumn{2}{c}{ IZQUIERDA } \\
& Encuesta & Votación & Encuesta & Votación & Encuesta & Votación \\
\hline & & & & & & \\
$1957 \mathrm{C}$ & 18.3 & 33.0 & 8.2 & 42.3 & 15.1 & 10.7 \\
$1958 \mathrm{P}$ & 31.4 & 31.2 & 17.7 & 35.7 & 24.3 & 28.6 \\
$1961 \mathrm{C}$ & 23.7 & 30.4 & 28.2 & 43.7 & 26.6 & 22.1 \\
$1964 \mathrm{P}$ & 15.0 & $* *$ & 23.6 & 60.6 & 23.4 & 38.6 \\
$1965 \mathrm{C}$ & 20.8 & 12.5 & 29.1 & 55.6 & 28.4 & 22.7 \\
$1967 \mathrm{M}$ & 21.1 & 14.3 & 21.8 & 51.8 & 33.3 & 28.7 \\
$1969 \mathrm{C}$ & 23.6 & 20.0 & 26.8 & 42.8 & 27.2 & 28.1 \\
$1970 \mathrm{P}$ & 23.7 & 34.9 & 15.7 & 27.8 & 32.7. & 36.2 \\
$1973 \mathrm{C}$ & 21.8 & 21.3 & 26.8 & 32.8 & 42.8 & 34.9 \\
$1989 \mathrm{C} *$ & 19.4 & 43.0 & 39.6 & 35.0 & 24.7 & 22.0 \\
\hline
\end{tabular}

* El cálculo para 1989 entre el centro y la izquierda es sólo aproximado. pues en general fueron en lista conjunta de votación indistinguible (Baño, 1990).

** En 1964 la derecha votó por el candidato demócratacristiano Eduardo Frei,

$\mathrm{C}=$ Congreso; $\mathrm{M}=$ Municipal; $\mathrm{P}=$ Presidencial

La explicación para ello podria estar no sólo en la relatividad del posicionamiento político. sino en el hecho que las elecciones se plantean en términos de partidos y líderes, y no de tendencias. A pesar de que, según vimos, la imagen de la posición de los partidos resulta clara para los entrevistados, las preferencias políticas no tienen una relación tan clara con la autoubicación en el espacio político. Situación que es particularmente clara en el caso de la derecha.

Todo esto pareciera estar dando cuenta de una extraña paradoja. Los sujetos conocen bien Y manejan a la perfección el espacio político como eje ordenador del sistema de partidos, al punto que son capaces de ubicarlos con precision aún después de quince años de exclusión de vida política partidaria. Sin embargo, los partidos que prefieren y los partidos que votan no muestran una estrecha relación con las posiciones políticas que declaran preferir personalmente. Situación que puede observarse antes del golpe militar de 1973 y que se presenta aún con mayor fuerza en los años de desenlace de la transición 1987-1989.

Esto nos lleva a examinar las actitudes de la población estudiada en relación con los partidos políticos. 


\section{LA APROXIMACIÓN A LOS PARTIDOS}

Una de las características más comunmente resaltadas en los estudios sobre el sistema político chileno es la importancia de los partidos (Valenzuela, 1989; Garretón, 1983: Sartori, 1980). Sin embargo. como señalamos anteriormente, ello no implica una buena imagen de los partidos. Tal situación se grafica perfectamente con los resultados del panel en cuanto percepciones sobre los partidos políticos que ya vimos. La imagen es en general mala, salvo en cuanto se les considera necesarios para representar intereses sociales, pero aún en esta aceptación mínima sólo participan dos tercios de los entrevistados. Carecemos de datos comparables para el período anterior al qolpe militar, salvo en que se les considera necesarios para gobernar en proporción similar a su aceptación en la transición como necesarios para representar intereses sociales. No parece aventurado sostener que en Chile, como en otros países, los partidos son aceptados como necesarios, pero no suscitan especiales simpatias. Si bien no tenemos con anterioridad datos, como los del panel, que tiendan a ratificar esta impresión, al menos tenemos que ante la altemativa de apoyar al candidato que gusta, pero que no pertenece al partido que se prefiere, o apoyar al candidato de ese partido. siempre se opta mayoritariamente por preferir al candidato por sobre el partido. Una medida indirecta de poco apego a los partidos.

Por otra parte, a pesar de ocupar un papel tan esencial, existe una muy baja afiliación o pertenencia a partidos. Las encuestas realizadas en el periodo 1986 a 1990 incluyeron en algunos casos preguntas sobre filiación partidaria, pero se obtuvieron porcentajes tan bajos, correspondientes por lo demás a los datos de los actuales registros públicos de adherentes, que se optó por eliminar la pregunta. Y no es éste un efecto de lenta repolitización, ya que en encuestas anteriores a 1973 se encuentra también una pertenencia a partidos bajísima.

Pero los partidos funcionaban y, al parecer, eran efectivamente centrales en el sistema político, fuertes electoralmente, permanentes y conformando un sistema que actuaba como eje de buena parte de la vida social, permeando prácticamente todas las organizaciones sociales de importancia. Aunque la gente no pertenecía a partidos. tenía que tener preferencias partidarias porque elegía de acuerdo a esas preferencias. Con la sola excepción del populismo ibañista de 1952. las elecciones a los principales cargos públicos se hacían entre los partidos.

Al examinar los datos sobre preferencias políticas de encuestas que van de 1958 a 1973 y el panel en sus tres olas, encontramos una fuerte proporción de personas que declaran tal proximidad. No obstante. hay que consignar que una proporción variable, pero que se acerca al tercio de los entrevistados, no se declara próximo a ningún partido (Cuadro №21). Además. pareciera haber una cierta relación entre no declarar proximidad a partidos y declararla a favor de partidos de centro, especialmente la Democracia Cristiana. Estoes, la disminución de los que no declaran proximidad se corresponde con el aumento de la preferencia por partido de centro (DC) y viceversa. Lo cual es una especie de ratificación, a nivel de partido, de la idea del «falso centro», es decir, que existe una proporción de entrevistados que, no teniendo una preferencia definida, optan por señalar la que aparece como menos contlictiva; el centro. Circunstancia que refuerza también las dudas acerca de la extensión social de las actitudes políticas.

En cuanto a la distribución de las declaraciones de proximidad a partidos políticos, ella parece tener una razonable relación con la historia electoral efectiva del país en los años correspondientes. El partido que aparece proporcionalmente más subrepresentado es el Partido Nacional. lo cual talvez se entiende de acuerdo a lo que se podría denominar «derecha no 
política». Fenómeno que convendría estudiar, puesto que pareciera surgir con extraordinaria fuerza al concluir el régimen autoritario, según se pudo apreciar no sólo en los datos del panel a la vista. sino en numerosas encuestas que estuvieron lejos de acertar la alta votación obtenida por el general Pinochet y la derecha política en el plebiscito y las elecciones generales, respectivamente.

\section{Cuadro $\mathrm{N}^{2} 21$}

Proximidad a Partidos/Partido Que gusta más

(PoRCENTAJES)

\begin{tabular}{|c|c|c|c|c|c|c|c|c|c|}
\hline & PC & $\mathrm{DC}$ & $\mathrm{PN}$ & $\mathrm{RN}$ & UDI & PR & PS & PPD & NS/NR Otros \\
\hline $1958 / 7$ & 3.7 & 13.5 & $21.4^{*}$ & - & . & 10.5 & 8.6 & - & 42.2 \\
\hline $1961 / 7$ & 4.7 & 16.8 & $18.8^{*}$ & - & - & 15.8 & 7.8 & - & 36.1 \\
\hline $1964 / 8$ & 2.8 & 34.4 & $5.4^{*}$ & - & - & 8.6 & 9.5 & - & 39.3 \\
\hline $1966 / 5$ & 1.9 & 51.8 & 3.8 & - & - & 6.3 & 8.0 & - & 28.1 \\
\hline $1967 / 2$ & 4.3 & 44.4 & 7.3 & - & - & 7.1 & 10.4 & - & 26.5 \\
\hline $1967 / 5$ & 7.7 & 50.2 & 9.7 & - & - & 6.7 & 11.0 & - & 14.4 \\
\hline $1967 / 11$ & 6.5 & 39.9 & 7.8 & - & - & 8.3 & 13.5 & - & 35.1 \\
\hline $1969 / 2$ & 7.6 & 35.4 & 11.3 & - & - & 5.0 & 12.3 & - & 28.4 \\
\hline $1969 / 4$ & 9.9 & 34.2 & 10.1 & - & - & 5.2 & 10.1 & - & 30.6 \\
\hline $1969 / 7$ & 6.1 & 29.4 & 14.2 & - & & 4.7 & 11.6 & - & 33.7 \\
\hline $1970 / 3$ & 5.1 & 29.6 & 11.5 & - & - & 6.2 & 11.6 & - & 35.8 \\
\hline $1970 / 3$ & 6.7 & 27.5 & 12.1 & - & - & 3.0 & 11.4 & - & 39.2 \\
\hline $1970 / 5$ & 6.5 & 289 & 18.0 & - & - & 5.2 & 17.3 & - & 24.2 \\
\hline $1970 / 7$ & 8.8 & 25.3 & 13.9 & - & - & - & 15.1 & - & 36.8 \\
\hline $1970 / 8$ & 4.6 & 23.9 & 11.0 & - & - & 3.3 & 15.4 & - & 41.9 \\
\hline $1970 / 8$ & 6.0 & 21.2 & 10.9 & - & - & 2.6 & 14.0 & - & 45.2 \\
\hline $1972 / 4$ & 7.6 & 21.3 & 7.2 & - & - & 0.6 & 25.7 & - & 37.7 \\
\hline $1972 / 4$ & 9.4 & 32.2 & 11.7 & - & - & 2.1 & 19.7 & - & 24.3 \\
\hline $1973 / 2$ & 9.9 & 32.1 & 9.2 & - & - & 1.6 & 24.1 & - & 23.0 \\
\hline $1987 / 11$ & 2.9 & 37.8 & 9.1 & 3.3 & - & 2.7 & 9.2 & - & 35.0 \\
\hline $1988 / 8$ & 3.1 & 34.7 & 5.7 & 4.6 & - & 2.4 & 8.0 & 6.7 & 34.8 \\
\hline $1989 / 11$ & 2.1 & 37.2 & 4.7 & 6.9 & 4.3 & 2.9 & 6.6 & 8.1 & 27.2 \\
\hline
\end{tabular}

* Se considera aquí el promedio de los partidos de derecha, Conservador y Liberal que luego se fusionan en el Partido Nacional.

Al parecer, la derecha, al plantearse como mantención de lo dado, se presenta como menos conflictiva y, al mismo tiempo, sus partidos tienen menos atractivos, ya que es más difícil plantearse la perspectiva de que haya que organizarse y hacer algo para que las cosas sigan como están, a menos que se perciba que hay una catástrofe que lo amenaza. Como señalamos antes, esto está ya en la formación del sistema de partidos modernos, que comienza con la formación de una organización de intención transformadora.

Esta situación ya se había manifestado en el hecho de que, en 1958 y 1970 , el candidato presidencial de la derecha. Jorge Alessandri, se tuviera que presentar como independiente para poder competir con éxito la primera vez y llegar muy cerca la segunda. 
En el período de transición 1987-89 se acentúa un fuerte rechazo a la políticá como conflicto provocado por quienes propugnan cambios drásticos. Lo político pasa a ser visto como esa intención de transformación conflictiva. La izquierda se define así claramente como política, mientras que el pensamiento de derecha no se define como político, sino como ajeno a ella, independiente, incluso neutral. No obstante, el pensamiento conservador vota por la derecha, dándole una fuerza que nunca tuvo en la historia reciente. Esa preferencia por la derecha se habría ocultado en las encuestas bajo el independentismo y el centrismo.

Por otra parte, la distribución de la proximidad a partidos en el período del panel, nos estaría dando cuenta de que el resurgimiento de la actividad partidaria tiende a descongelar (Lipset y Rokkan. 1967) el sistema de partidos. Aunque aparece un marcado centrismo, volcado totalmente a la Democracia Cristiana, hay signos evidentes de reacomodo tanto en la izquierda como en la derecha. En la izquierda la aparición del PPD (introducido en la segunda ola del panel entre las alternativas) compite claramente con el antiguo Partido Socialista, mientras que los comunistas se ven en franca declinación que amenaza su existencia. A su vez, en la derecha, Renovación Nacional inicia el fuerte crecimiento que terminaría por desplazar al antiguo Partido Nacional, mientras que la UDI (introducida solo en la tercera ola) rápidamente entra a disputar el espacio de la derecha a Renovación Nacional.

Todos estos datos de proximidad a partidos, especialmente al momento de la transición, tienden a concordar con los análisis que se hacen del proceso político chileno. Sin embargo, ello acontece sólo si se les toma de manera muy genérica y saltándose gruesas incongruencias, como es el caso de subrepresentaciones o sobrepresentaciones notorias. Marcan tendencias que son más ajustadas que el posicionamiento político, pero se mantienen diferencias importantes con lo que es la decisión política efectiva del voto. Hay ciertas vaguedades en la aproximación que permanecen sin entenderse. Lo mismo ocurre con ese tercio de entrevistados que no declaran proximidad a partido y que, sin embargo. terminan por decidir electoralmente entre ellos.

Como era de esperar, la proximidad a partidos, al igual que la autoubicación en el espacio político, da cuenta también del aumento del compromiso político y de la agudización del conflicto a comienzos de la década del setenta. Se ratifica así no sólo el enfrentamiento de aquella época, sino que la extensión social de tal enfrentamiento. A la vez, nos señala el período de transición 1987-1989 como el menos conflictivo.

También aquí tenemos evidencias de lo que denominamos "oportunismo político». Es notorio que en vísperas de las elecciones presidenciales de septiembre de 1958, 1964 y 1970. aumenta la proporción de personas que «se agachan», esto es que no declaran proximidad ni gusto por ningún partido. Además, hay una cierta tendencia a que los partidos triunfantes en elecciones presidenciales reciban fuerte apoyo despućs de ese triunfo. Al menos eso es claro despues del triunfo DC en 1964 y el de izquierda en 1970.

En el período 1987-1989 hay variaciones en este comportamiento oportunista. Se mantienen los que no se declaran próximos a ningún partido en 1988, antes del plebiscito presidencial. pero habría un considerable aumento si es que no se introduce la nueva opción PPD. En cambio, antes de las elecciones generales hay una disminución de los no comprometidos, lo cual podría entenderse en términos deI clima de distensión que se produjo luego que las FF AA y el gobierno aceptaran la derrota del general Pinochet en el plebiscito de 1988.

Después de todo, la elección del partido que se reconoce como más próximo o que más gusta, ratifica las consideraciones que se tenían a partir del posicionamiento político, pero no 
aclaran las diferencias que se encuentran entre ambas clasificaciones y mucho menos permiten entender cómo opera el proceso de decisión, que permite que la votación efectiva se separe de las previsiones que se pudieran hacer a partir del partido preferido y la autoubicación en el espacio político.

Por cierto que debemos recordar que un elemento importante para entender estas variaciones se encuentra en el hecho de que, según hemos visto más atrás, un sector importante de la población pareciera no tener una actitud previamente definida. Pero, si descartamos a aquellos sujetos que le dan estabilidad a la actitud, dentro de los cuales se encuentran no sólo los firmemente definidos, sino que también aquellos firmemente no definidos, pues estos últimos optarán establemente según el cálculo de probabilidades, tenemos a aquellos sujetos que se definirán por la contingencia.

De ser muy numeroso el conjunto de los que se definen por la contingencia, tendríamos un elevado grado de inestabilidad política, lo cual se expresaría en importantes variaciones tanto en el posicionamiento político como en las preferencias partidarias. Dado que no se presentan grandes variaciones, se puede especular que, a pesar de la poca estabilidad de las actitudes individuales, se produce una estabilidad social derivada precisamente de la inexistencia 0 debilidad de las actitudes.

Por otra parte, la diferencia que se observa entre la actitud declarada y la votacion efectiva pareciera deberse a un elevado grado de indefinición del posicionamiento político, especialmente en el centro, con el que buscan identificarse los partidos de derecha. Al mismo tiempo, la independencia e indefinición política se asocia a los partidarios del regimen militar que explícitamente se declara no político. lo cual lleva a que esa no definición se traduzca en votación favorable a la derecha.

\section{BIBLIOGRAFÍA CITADA}

A.DUnate, A., 1985, Antecedentes socioeconómicos y resultados electorales, Documento de Trabajo, FLACSO, Chile.

BANO, R. 1989, Estabilidad y predictividad de las orientaciones politicas. Documento de Trabajo FLACSO.

3ațo. R., 1990, La gente piensa algo sobre los partidos. Documento de Trabajo. FL.ACSO, Chile.

3.র0. R., 1990, «Elecciones en Chile, ¿otra vez lo mismo o al revés», Revista Española de Investigaciones Sociológicas $\mathrm{N}^{\circ} 50$, Abril-Junio, Madrid.

Bato. R. y CANales, M., 1991, El agitado y raro camino a la democracia. Chile en los años 80. CEDEAL. Madrid. 3año, R. y Faletto, E., 1993, «Estructura social y estilo de desarrollo». Revista CEPAL № 50, Santiago.

CAłfPERo, G., 1987, «Los cambios en la estructura social» en Margen, Revista de Filosofía y Letras, Marzo, Santiago.

ERRONI, U., 1971, «Para una teoría del partido político» en Teoría Marxista del Partido Político, Ediciones Pasado $\because$ Presente, Argentina.

ONverse. Philip, 1964, "The nature of belief sistems in Mass Publics». en Arter, DAvD, Ideology and Discontent. The Free Press of Glencoe, London. 
REVISTA DE SOCIOLOGIA

FALETTO. E. 1980. Algunas características de la base social del partudo soctalista y del partido comunista. Documento de Trabajo $N^{0} 97$. FLACSO. Chile.

GARRETón, M. A., 1983, El proceso político chileno, FLACSO, Chile.

Lipset, S. y Rokkan, S., 1967, Party Systems and Voter Alignements. The Free Press, New York.

Martinez, J. y TIRoni, E. 1985, Las clases sociales en Chile: cambio y estratificación. 1970-1980 SUR.

MARTtNeZ, J. Y LEÓN, A., 1987, Clases y clasificación sociales, CED-SUR.

Sartori, G., 1980, Partidos y sistemas de partidos, Alianza Editorial.

Valenzuela, A., 1989, El quiebre de la democracia en Chile, FLACSO. Chile. 